\title{
Should macroeconomic forecasters use daily financial data and how?*
}

\author{
Elena Andreou ${ }^{\dagger} \quad$ Eric Ghysels ${ }^{\ddagger} \quad$ Andros Kourtellos ${ }^{\S}$
}

First Draft: May 2009

This Draft: November 18, 2009

\begin{abstract}
There are hundreds of financial times series available on a daily basis that contain information about the future states of the economy. Can we efficiently use all this daily financial information for improving and/or updating macroeconomic forecasts? The literature on macroeconomic forecasting has not address this question. Instead, it has focused on how the use of a small set of financial series - usually aggregated at a monthly or quarterly frequency. In the paper we introduce two methods for predicting inflation and real activity: (1) methods which rely on combinations of regressions that involve regressors with different sampling frequency, such as quarterly macro series and daily financial series and (2) a small set of daily financial factors extracted from the large cross-section of daily series along with quarterly frequency factors dominated by macroeconomic variables. Both methods have the following important features: (1) they allow us to clearly show the incremental value of daily financial series in terms of forecast improvements, (2) they provide a succinct summary of a huge amount of information in daily financial data, (3) they rely on extremely simple parsimonious regression methods that are easy to implement in practice. The analysis of daily financial factors are of independent interest and other potential applications too as they span equity premium, foreign exchange, fixed income and commodity price information.
\end{abstract}

*The second author benefited from funding by the Federal Reserve Bank of New York through the Resident Scholar Program. We would like to thank Tobias Adrian, Jennie Bai, Jushan Bai, Frank Diebold, Rob Engle, Ana Galvão, Michael Fleming, Serena Ng, Simon Potter, Lucrezia Reichlin, Jim Stock, Mark W. Watson as well as seminar participants at Columbia University, the Federal Reserve Bank of New York, MEG Conference, Queen Mary University, the University of Pennsylvania and the CIRANO/CIREQF Financial Econometrics Conference, the NBER Summer Institute, and the Deutsch Bundesbank for comments. We also thank Constantinos Kourouyiannis and Michael Sockin for providing excellent research assistance.

${ }^{\dagger}$ Department of Economics, University of Cyprus, P.O. Box 537, CY 1678 Nicosia, Cyprus, e-mail: elena.andreou@ucy.ac.cy.

${ }^{\ddagger}$ Department of Economics, University of North Carolina, Gardner Hall CB 3305, Chapel Hill, NC 275993305, USA, and Department of Finance, Kenan-Flagler Business School, e-mail: eghysels@unc.edu.

${ }^{\S}$ Department of Economics, University of Cyprus, P.O. Box 537, CY 1678 Nicosia, Cyprus, e-mail: andros@ucy.ac.cy 


\section{Introduction}

There are literally hundreds of financial times series available on a daily basis that contain information about the future states of the economy. Can we use them for the purpose of improving and/or updating macroeconomic forecasts? The literature on macroeconomic forecasting has not address this question. Instead, it has focused on how the use of a small set of financial series - usually aggregated at a monthly or quarterly frequency - to improve macroeconomic forecasts. Since macroeconomic data are typically sampled at low frequency, that is quarterly or monthly, the standard approach is to match such data with monthly or quarterly aggregates of financial data to build prediction models for the macro economy. For example, typically one does not use a daily measure of the slope of the term structure, but instead a quarterly or monthly average. Such aggregation often implies loss of information which translates to forecasting losses. Moreover, the low frequency nature of macroeconomic data releases creates another difficulty. For example, GDP growth is announced with considerable delay and is only released every quarter. Monthly series, like inflation, or industrial production are also announced with at least one month delay. Similarly while economic theory suggests that the forward looking behavior of financial asset prices should be considered as good predictors for economic conditions, the empirical evidence based on quarterly and monthly financial data is mixed and not robust (for example see Stock and Watson (1989), Stock and Watson (2002), Forni, Hallin, Lippi, and Reichlin (2000) and Forni, Hallin, Lippi, and Reichlin (2003)).

In the paper we introduce two types of prediction methods for inflation and real activity: (1) methods which rely on combinations of regressions that involve regressors with different sampling frequency, such as quarterly macro factors and daily financial series and (2) a small set of daily financial factors extracted from the large cross-section of daily series along with quarterly frequency factors dominated by macroeconomic variables. Both methods have the following important features: (1) they allow us to clearly show the incremental value of daily financial series in terms of forecast improvements, (2) they provide a succinct summary of a huge amount of information in daily financial data, (3) they rely on extremely simple parsimonious regression methods that are easy to implement in practice. The daily financial factors - what happen to find about 10 of them that summarize the cross-sectionare of independent interest as they span equity premium, fixed income, commodity price information. 
We employ regression models that involve data sampled at different frequencies, the so called Mi(xed) Da(ta) S(ampling), or MIDAS, regression models. MIDAS was introduced in both a forecasting and regression context in a number of recent papers, including recent work by Ghysels, Santa-Clara, and Valkanov (2002), Ghysels, Santa-Clara, and Valkanov (2006) and Andreou, Ghysels, and Kourtellos (2008). Such regressions have been used in the context of improving quarterly macro forecasts with monthly data (see e.g. Armesto, Hernandez-Murillo, Owyang, and Piger (2008), Clements and Galvão (2008a), Clements and Galvão (2008b), Galvão (2006), Schumacher and Breitung (2008), Tay (2007) ), or improving quarterly and monthly macroeconomic predictions with daily financial data (see e.g. Monteforte and Moretti (2008), Ghysels and Wright (2008), Hamilton (2006), Tay $(2006))$.

Our results provide some interesting findings for forecasting inflation and economic activity for the period 1999-2008. We find that the best MIDAS models provide substantial forecasting gains relative to the benchmark forecasting models considered in the literature. More importantly, the best MIDAS models improve substantially the forecasts of the best traditional models that use quarterly lags of averaged daily data to forecast CPI and PCE inflation, IP and GDP growth. Interestingly, we find that the top percentile of best predictors for $\mathrm{h}=1$ and $\mathrm{h}=4$ comprise commodity prices and interest rate spreads while the best models are generally given by MIDAS-ADL with no quarterly factors. Moreover, daily information of financial indicators like the daily Breakeven inflation, crude oil prices, noncommercial paper spread and 1 month libor contain useful information for predicting CPI and PCE inflation. In the case of GDP growth we find the best predictors include daily Canadian/US Dollar returns and the daily Aruoba, Diebold and Scotti (ADS) indicator for $\mathrm{h}=1$ and interest rate spreads for $\mathrm{h}=4$. In contrast to the case of forecasting CPI and PCE inflation, we find that models with quarterly factors are important for forecasting GDP growth. Finally, the daily factors appear to have useful information for forecasting inflation and economic activity beyond the information included in the quarterly factors. MIDAS models can efficiently incorporate this information and provide accurate forecasts, especially when these models incorporate real-time information using daily leads within the quarter.

The paper is organized as follows. In section 2 and 3 we describe the MIDAS Regression Models and discuss our data. In section 4 we present our results and section 5 concludes. 


\section{MIDAS Regression Models}

We are interested in forecasting at a quarterly horizon, namely one quarter ahead up to four quarters, a series denoted $Y_{t}^{Q}$. The time index $t$ will refer to the quarterly frequency of forecast horizons. The typical Augmented Distributed Lag, $A D L\left(p_{Y}^{Q}, q_{X}^{Q}\right)$, model is:

$$
Y_{t+1}^{Q}=\mu+\sum_{k=0}^{p_{Y}^{Q}-1} \alpha_{k} Y_{t-k}^{Q}+\sum_{k=0}^{p_{X}^{Q}-1} \beta_{k} X_{t-k}^{Q}+u_{t+1}
$$

as it involves $p_{Y}^{Q}$ lags of $Y_{t}^{Q}$ and $q_{X}^{Q}$ lags of a regressor sampled also at a quarterly frequency. This regression is fairly parsimonious as it only requires $p_{Y}^{Q}+q_{X}^{Q}+1$ parameters to be estimated. One such example in the literature is the case where $Y_{t+1}^{Q}$ represents quarterly inflation and $X_{t-k}^{Q}$ represents interest rate yields.

In many situations we do have monthly, weekly or daily data available for regressors. We will denote them respectively $X_{j, t}^{M}, X_{j, t}^{W}$, or $X_{j, t}^{D}$, with $j$ referring to the $j^{\text {th }}$ month, week or day in quarter $t$. An illustrative example is the prediction of (quarterly) inflation with a spot commodity price as regressor. While we can formulate equation (2.1) using past quarterly inflation and past (say quarterly averaged) commodity prices, we could also consider:

$$
Y_{t+1}^{Q}=\mu+\sum_{k=0}^{p_{Y}^{Q}-1} \alpha_{k} Y_{t-k}^{Q}+\sum_{j=1}^{N_{D}} \beta_{j} X_{j, t}^{D}+u_{t+1}
$$

assuming for simplicity that we take one quarter of daily data, namely $N_{D}$ lags (number of trading days per quarter - we assume it to be constant for simplicity). Take again the case of lags that amount to one past quarter's information. In such a case we have $\left(N_{D}+1+1\right)$ parameters to estimate. When, say $N_{D}=66$, we have a total of $66+p_{Y}^{Q}+1$ parameters. This illustrative parameter proliferation problem example also explains why high frequency data has been avoided.

We develop two strategies to address the use of high frequency financial data for forecasting key macroeconomic variables. One involves the use of MIDAS regressions with a single high frequency regressor - using a cross-section of daily financial series- and then combines the forecasts they generate. The second involves extracting factors from two large cross-sections that involve quarterly data and daily financial data. The latter approach involves extracting 
financial factors that span many series within the equities, foreign exchange, fixed income and commodity prices. These daily financial factors can be used for many other applications beyond the present forecasting analysis.

One appealing approach is to solve parameter proliferation by using MIDAS regression models. The main idea - which is rather simple and fairly straightforward to implement - is to hyper-parameterize the lag coefficient weights. Hence, one can rewrite equation (2.2) to define $A D L-M I D A S\left(p_{Y}^{Q}, k_{X}^{D}\right)$

$$
Y_{t+1}^{Q}=\mu+\sum_{k=0}^{p_{Y}^{Q}-1} \alpha_{k} Y_{t-k}^{Q}+\beta \sum_{j=0}^{k_{X}^{D}-1} \sum_{i=1}^{N_{D}} w_{i+j * k_{X}^{D}}\left(\theta_{X}^{D}\right) X_{i, t-j}^{D}+u_{t+1},
$$

where $k_{X}^{D}$ is a multiple of the number of trading days in a quarter, $N_{D}$. Allowing daily lags beyond the past quarter allows ADL-MIDAS to model persistent memory structures in a very parsimonious way.

Following Ghysels, Santa-Clara, and Valkanov (2006) and Ghysels, Sinko, and Valkanov (2006), we employ a two parameter exponential Almon lag polynomial

$$
w_{j}\left(\theta_{1}, \theta_{2}\right)=\frac{\exp \left\{\theta_{1} j+\theta_{2} j^{2}\right\}}{\sum_{j=1}^{m} \exp \left\{\theta_{1} j+\theta_{2} j^{2}\right\}}
$$

with $\theta=\left(\theta_{1}, \theta_{2}\right)$. This approach allows us to obtain a linear projection of high frequency data $X_{t}^{D}$ onto $Y_{t}^{Q}$ with a small set of parameters.

At this point several issues emerge. Some issues are theoretical in nature. For example, to what extend is this tightly parameterized formulation in (2.3) able to approximate the unconstrained (albeit practically infeasible) projection in equation 2.2]? There is also the question whether a more traditional approach involving the Kalman filter would be more suitable We do not deal directly with these types of questions here, as they have been addressed notably in Bai, Ghysels, and Wright (2009) and Kuzin, Marcellino, and Schumacher (2009). However, some short answers to these questions are as follows. First, it turns out that a MIDAS regression can be viewed as a reduced form representation of the linear projection that emerges from a state space model approach - by reduced form we mean

\footnotetext{
${ }^{1}$ The mismatch of sampling frequency has been address in the context of state space models by Harvey and Pierse (1984), Harvey (1989), Bernanke, Gertler, and Watson (1997), Zadrozny (1990), Mariano and Murasawa (2003), Mittnik and Zadrozny (2004), Aruoba, Diebold, and Scotti (2009), Ghysels and Wright (2008), Kuzin, Marcellino, and Schumacher (2009), among others.
} 
that the MIDAS regression does not require the specification of a full state space system of equations. In some cases the MIDAS regression is an exact representation of the Kalman filter, in other cases it involves approximation errors that are typically small $]^{2}$ The Kalman filter, while clearly optimal as far as linear projections goes, has two main disadvantages (1) it is more prone to specification errors as a full system of equations for $Y, X$, and latent factors is required and (2) as a consequence it typically requires a lot more parameters to achieve the same goal. This is particularly relevant for the cases we cover in this paper. Namely handling a combination of quarterly and daily data leads to large state space system equations prone to mis-specification. MIDAS regressions, in comparison, are frugal in terms of parameters and achieve the same goal. More parameters and a system of equations also means that estimation is more numerically involved - something that is not so appealing when dealing with large data sets - as we will. In the remainder of this section we expand on the main theme addressed so far. Namely, we will present several MIDAS regression specifications that cover more general cases.

\subsection{Temporal aggregation and multiplicative MIDAS regressions}

It is worth pointing out that there is a more subtle relationship between the ADL regression appearing in equation (2.1) and the ADL-MIDAS regression in equation (2.3). Note that the ADL regression involves temporally aggregated series, based for example on equal weights of daily data, i.e

$$
X_{t}^{Q} \equiv\left(X_{1, t}^{D}+X_{2, t}^{D}+\ldots+X_{N_{D}, t}^{D}\right) / N_{D}
$$

If we take the case of $N_{D}$ days of past daily data in an ADL regression, then implicitly through the aggregation we have picked the weighting scheme $\beta_{1} / N_{D}$ for the daily data $X_{., t}^{D}$. We will sometimes refer this scheme as a flat aggregation scheme ${ }^{3}$ While these weights might be "natural" for purposes of temporal aggregation, it may not be optimal for the purpose of forecasting as more recent data may probably get more weight. In some sense

\footnotetext{
2 Bai, Ghysels, and Wright (2009) discusses both the cases where the mapping is exact and the approximation errors in cases where the MIDAS does not coincide with the Kalman filter.

${ }^{3}$ Note that we could define $X_{t}^{Q}$ with the number of lags larger than $N_{D}$. This more general setting is not considered here. The appeal of using $N_{D}$ lags is that (1) it simplifies the structure of the models, (2) directly relates to temporal aggregation and (3) maps into the Kalman filter.
} 
the ADL-MIDAS regression lets the data decide what those weights should be.

The comparison with temporal aggregation prompts us to consider two MIDAS regression models that allow for quarterly lags. First, define the following filtered parameter-driven quarterly variable

$$
X_{t}^{Q}\left(\theta_{X}^{D}\right) \equiv \sum_{i=1}^{N^{D}} w_{i}\left(\theta_{X}^{D}\right) X_{i, t}^{D}
$$

Then, we can define the $A D L-M I D A S-M\left(p_{Y}^{Q}, p_{X}^{Q}\right)$ model, where $-M$ refers to the fact that the model involves a multiplicative weighting scheme, namely:

$$
Y_{t+1}^{Q}=\mu+\sum_{k=0}^{p_{Y}^{Q}-1} \alpha_{k} Y_{t-k}^{Q}+\sum_{k=0}^{p_{X}^{Q}-1} \beta_{k} X_{t-k}^{Q}\left(\theta_{X}^{D}\right)+u_{t+1}
$$

and $A D L-M I D A S-M\left(p_{Y}^{Q}[r], p_{X}^{Q}[r]\right)$ model:

$$
Y_{t+1}^{Q}=\mu+\alpha \sum_{k=0}^{p_{Y}^{Q}-1} w_{k}\left(\theta_{Y}^{Q}\right) Y_{t-k}^{Q}+\beta \sum_{k=0}^{p_{X}^{Q}-1} w_{k}\left(\theta_{X}^{Q}\right) X_{t-k}^{Q}\left(\theta_{X}^{D}\right)+u_{t+1}
$$

Both equations 2.5 and (2.6) apply MIDAS aggregation to the daily data of one quarter but they differ in the way they treat the quarterly lags. More precisely, while equation (2.5) does not restrict the coefficients of the quarterly lags, equation (2.6) restricts the coefficients of the quarterly lags - hence the notation $p_{X}^{Q}[r]$ - by hyper-parameterizing these coefficients using a multiplicative MIDAS polynomial. ${ }^{4}$ Both specifications nest the equally weighted aggregation scheme. The multiplicative structure in 2.5) and (2.6) has certain advantages, namely Bai, Ghysels, and Wright (2009) show that the weighting scheme in equation (2.5) corresponds to the structure of a steady state Kalman filter linear projection with mixed sampling frequencies. The downside of the MIDAS specification in equation (2.5) is that it is less parsimonious than the single weighting scheme in equation (2.3). On the other hand, equation (2.6) is more parsimonious than equation (2.5), and at the same time also more restrictive.

\footnotetext{
${ }^{4}$ The multiplicative MIDAS scheme was originally suggested for purpose of dealing with intra-daily seasonality in high frequency data, see Chen and Ghysels (2009).
} 


\section{$2.2 \quad$ MIDAS Regression Models with Factors}

Recently, a large body of recent work has developed factor model techniques that are tailored to exploit a large cross-sectional dimension; see for instance, Bai and Ng (2002), Bai (2003), Forni, Hallin, Lippi, and Reichlin (2000), Forni, Hallin, Lippi, and Reichlin (2005), Stock and Watson (1989), Stock and Watson (2003), among many others. These factors are usually estimated at quarterly frequency using a large cross-section of time-series. Following this literature we investigate first whether we can improve factor model forecasts by augmenting such models with high frequency information, especially daily financial data. Subsequently, we will construct daily factors, using the large cross-section of financial series.

We augment the aforementioned MIDAS models with factors, $F_{t}$, obtained by following factor model

$$
\begin{aligned}
X_{t} & =\Lambda_{t} F_{t}+u_{t} \\
F_{t} & =\Phi F_{t-1}+\eta_{t} \\
u_{i t} & =a_{i t}(L) u_{i t-1}+\varepsilon_{i t}, \quad i=1,2, \ldots, n .
\end{aligned}
$$

The data used to implement the factor representation will be described in the next section. Suffice it here to say that we use series similar to those used by Stock and Watson (2008). The number of factors are chosen based on the information criteria proposed by Bai and $\mathrm{Ng}$ (2002).

We augment the MIDAS regression models from the previous subsection by adding quarterly factors. For instance, equation (2.3) generalizes to the $F A D L-M I D A S\left(p_{Y}^{Q}, p_{F}^{Q}, k_{X}^{D}\right)$ model

$$
Y_{t+1}^{Q}=\mu+\sum_{k=0}^{p_{Y}^{Q}-1} \alpha_{k} Y_{t-k}^{Q}+\sum_{k=0}^{p_{F}^{Q}-1} \beta_{k} F_{t-k}^{Q}+\gamma \sum_{j=0}^{k_{X}^{D}-1} \sum_{i=1}^{N_{D}} w_{i+j * k_{X}^{D}}\left(\theta_{X}^{D}\right) X_{i, t-j}^{D}+u_{t+1}
$$

Note that we can also formulate a $F A D L-M I D A S-M\left(p_{Y}^{Q}, p_{F}^{Q}, p_{X}^{Q}\right)$ model, which involves the multiplicative MIDAS weighting scheme, hence generalizing equation 2.5). Notice also that equation (2.8) simplifies to the traditional factor model with additional regressors when the MIDAS features are turned off - i.e. say a flat aggregation scheme is used. When the lagged dependent variable is excluded then we have a projection on daily data, combined with 
aggregate factors. This brings us to the following benchmark models of $F A D L\left(p_{Y}^{Q}, p_{F}^{Q}, p_{X}^{Q}\right)$

$$
Y_{t+1}^{Q}=\mu+\sum_{k=0}^{p_{Y}^{Q}-1} \alpha_{k} Y_{t-k}^{Q}+\sum_{k=0}^{p_{F}^{Q}-1} \beta_{k} F_{t-k}^{Q}+\sum_{k=0}^{p_{X}^{Q}-1} \gamma_{k} X_{t-k}^{Q}+u_{t+1}
$$

and $F A R\left(p_{Y}^{Q}, p_{X}^{Q}\right)$ when the regressor $X^{Q}$ is not present

$$
Y_{t+1}^{Q}=\mu+\sum_{k=0}^{p_{Y}^{Q}-1} \alpha_{k} Y_{t-k}^{Q}+\sum_{k=0}^{p_{F}^{Q}-1} \beta_{k} F_{t-k}^{Q}+u_{t+1}
$$

Finally, we consider model selection in the traditional setting, i.e. with respect to the choice between autoregressive (same frequency) models versus factor models or both combined. We consider, between zero and four quarterly (low frequency) lags, $p_{Y}$, of $Y_{t}\left(\theta_{Y}^{M}\right)$ and between one and four quarterly lags, $q_{X}$, of $X_{t}\left(\theta_{X}^{D}\right)$ and $F_{t}^{Q}$. In terms of the daily lags we consider the following multiples of the number of trading days, and $k_{X}^{D}=1,2,3,4$. We estimate the models with fixed lags but we also use AIC to select the number of quarterly and/or daily lags.

\subsection{Nowcasting and Leads}

Giannone, Reichlin, and Small (2008), among others, have formalized the process of updating the nowcast and forecasts as new releases of data become available. This process can be mimicked via MIDAS regression models with leads. Say we are one or two months into quarter $t+1$. Namely, we consider the MIDAS models with leads in order to incorporate real-time information available mainly on financial variables. Our objective is to forecast quarterly economic activity and in practice we often have a monthly release of macroeconomic data within the quarter and the equivalent of at least 44 trading days of financial data observed with no measurement error. This means that if we stand on the first day of the last month of the quarter and wish to make a forecast for the current quarter we could use and around 44 leads of daily data for financial markets that trade on weekdays.

Consider the Factor ADL model with MIDAS in equation 2.8, which allows for $J_{X}^{D}$ daily leads for the daily predictor, expressed in multiples of months, $J_{X}^{D}=1,2, \ldots, J$. Then we can 
specify the $F A D L-M I D A S\left(p_{Y}^{Q}, p_{F}^{Q}, k_{X}^{D}, J_{X}^{D}\right)$ model

$$
Y_{t+1}^{Q}=\mu+\sum_{k=0}^{p_{Y}^{Q}-1} \alpha_{k} Y_{t-k}^{Q}+\sum_{k=0}^{p_{F}^{Q}-1} \beta_{k} F_{t-k}^{Q}+\gamma \sum_{j=0}^{k_{X}^{D}-1} \sum_{i=1}^{N_{D}} w_{i+j * k_{X}^{D}}\left(\theta_{X}^{D}\right) X_{i+J_{X}^{D}, t-j}^{D}+u_{t+1}
$$

\section{$3 \quad$ Daily and Quarterly Factors and Data}

We forecast the US quarterly inflation rate and the growth rate of economic activity using various measures. For inflation we use monthly Consumer Price Index (CPI) and Core inflation (CPILFESL). For economic activity we use monthly Industrial Production (IP) and quarterly Real Gross Domestic Product (RGDP). We use two sample periods of US data for the post great moderation period: 1984:Q1-2008:Q4 (T=100) and 1999:Q1-2008:Q4 $(\mathrm{T}=40)$. The two subperiods involve different numbers, $\mathrm{N}$, of daily financial predictors due to daily data availability. We chose to focus on two post samples in the post Great Moderation period because this period appears to mark a structural change in many US macroeconomic variables (Stock and Watson, 2008, van Dijk and Sensier, 2004) and it is also documented that it is relatively difficult to predict key macroeconomic variables vis-a-vis the pre-1985 period and vis-a-vis simple univariate models such as the RW or AO models. The relatively shorter sample enables us to examine the role of a larger cross-section of daily financial predictors in improving macroeconomic forecasts in the last two decades.

For the longer sample starting from the mid 1980s, we estimate our models using the period 1986:Q1-1997:Q1 while forecasts are obtained for the period 1997:Q2-2008:Q4. For the shorter sample the estimation and forecasting windows are given by 1999:Q1-2005Q4 and 2006Q1-2008Q4, respectively. Using a recursive estimation method we provide pseudo outof-sample forecasts (see for instance, Stock and Watson, 1993) to evaluate the predictive ability of our models for various forecasting horizons $h=1,2,4$. For each model we obtain the root MSFE:

$$
R M S F E_{i, t}=\sqrt{\frac{1}{t-T_{0}+1} \sum_{\tau=T_{0}}^{t}\left(y_{\tau+h}^{h}-\widehat{y}_{i, \tau+h \mid \tau}^{h}\right)^{2}}
$$

where $t=T_{1}, \ldots, T_{2} . \quad T_{0}$ is the point at which the first individual pseudo out-of sample forecast is computed. Note that $T_{0}=2006: Q 1, T_{1}=2006: Q 1+h$, and 
$T_{2}=2008: Q 4-h$.

For conciseness we present the results of the shorter sample. We use two databases of two sampling frequencies of macroeconomic and financial indicators. The first is a quarterly dataset of 69 quarterly series of real output and income, capacity utilization, employment and hours, price indices, money e.t.c., described in detail in the Appendix, to extract the quarterly factors. This dataset excludes variables observed at the daily frequency and included in the daily dataset. The second is a daily dataset with large cross-section of 988 daily series for the recent period of 1999-2008 and a relative smaller cross-section of for four categories of financial assets: (i) the class of Commodities includes 241 variables such as US individual commodity prices, commodity indices and futures; (ii) the class of Corporate Risk includes 210 variables such as yields such as bonds for various maturities, libor, Certificate of Deposits, Eurodollars, Commercial Paper, default spreads using matched maturities, quality spreads, and other short term spreads such as TED; (iii) the class of Equities comprises 219 variables of the major international stock market returns indices and Fama-French factors and portfolio returns as well as US stock market volume of indices and option volatilities of market indices; (iv) the class of the Foreign Exchange Rates includes 70 variables of the trading partners in the broad index as well as international currency rates of major indices and effective exchange rate indices. (v) Government Securities include 248 variables of government treasury bonds rates and yields, term spreads, TIPS yields, break-even inflation. All variables are described in the Appendix.

Using these datasets we do three things: (1) extract quarterly factors; (2) extract daily factors; (3) investigate the predictive ability of a subset of the 988 predictors (90 variables) that have been proposed in the literature as the most important predictors for inflation and economic activity.

The tables in the Appendix refer to the variables names, short description and transformations. The data source for the quarterly and daily series are the FRB and Haver Analytics, a data warehouse that collects the data series from their individual sources (such as the Federal Reserve Board (FRB) to Chicago Board of Trade (CBOT) and others), the Global Financial Database (GFD) and FRB, unless otherwise stated.

Following the methodology of Stock and Watson (2008) we estimate Dynamic Factor models to construct the factors. The series were transformed in order to eliminate trends by first differencing (in many cases after taking logarithms as reported in the Appendix). There 
are alternative approaches of choosing the number of factors. One approach is to use the information criteria (ICP) proposed by Bai and Ng (2002). In the case of quarterly factors ICP criteria suggest the choice of the first two factors. This result is consistent with the finding of Stock and Watson (2008). Interestingly, although our quarterly factors differ from the Stock and Watson factors by not including the series for which daily information is available, our first two factors highly correlate with those of Stock and Watson. This suggests that the excluded aggregated daily series were not important for the first two factors. In the case of daily factors, however, ICP criteria are not helpful because they always suggest 10 factors and therefore, we study all 10 daily factors one at a time. Table 1 shows the $\%$ variation explained by each daily factor as well as how much load each class of assets provides to each daily factor. The latter is helpful to attach a label to each daily factor. For

instance, the first daily factor $F_{1}^{D}$ can be characterized as Government Securities while $F_{2}^{D}$ as Equities.

\section{Empirical results}

Table 3 presents the RMSFEs of the benchmark models for CPIall and PCEall inflation, namely the UCSV and AO models, as well as the RW model, which the literature adopts as the benchmark model for GDP and IP growth. In addition, Table 3 provides the summary results of the relative RMSFEs for the following categories of models: univariate AR models, traditional FAR models, (F)ADL and (F)-ADL-MIDAS models with individual daily predictors with and without quarterly factors, $\mathrm{F}$ as well as (F)ADL-DF and (F)-ADLMIDAS-DF models, which are the same as the latter two categories with daily factors (instead of daily predictors). The main message from Table 3 is that best MIDAS models (with the lowest RMSFE) provide substantial forecasting gains relative to the benchmark forecasting models considered in the literature, such as the UCSV, AO, RW, AR and FAR, during the last ten years of US data. In particular, comparing best MIDAS models with the traditional models that use quarterly lags of averaged daily data to forecast CPIall, PCEall and PCEcore inflation, as well as IP and GDP growth, MIDAS models improve around 45\%, 40\%, 45\%, $40 \%$, and $35 \%$ of the RMSFE of the UCSV, AO and RW benchmark models, respectively, for 1 and especially for 4 quarters ahead. Moreover, it is for 4 quarters ahead that MIDAS models for forecasting CPIall inflation and GDP growth, yield substantial gains vis-a-vis the traditional (F)ADL models that impose a flat aggregation scheme for the daily data. Let 
us first focus on the results of CPIall and PCEall with no leads presented in Table 3 . We find that the best models (with the lowest RMSFE across all models) for both variables are ADL-MIDAS models with relative RMSFE of $43 \%$ and $38 \%$ of the UCSV for both $h=1$ and 4 , as well as forecasting gains over the traditional ADL of around $35 \%$ and $65 \%$ for $h=1$ and 4 , respectively. Similar results are found for real GDP growth forecasts. The model with the lowest RMSFE for predicting GDP growth is the FADL-MIDAS model with relative gains of $37 \%$ and $44 \%$, for both $h=1$ or 4 , vis-a-vis the RW models, respectively. Introducing additional information regarding the daily leads of financial variables we find that the relative RMSFE of MIDAS models substantially improve forecasts in the two inflation core measures, CPIcore and PCEcore. It also interesting to compare these forecasting gains of MIDAS based on individual daily predictors with the best models which use daily financial factors.

In Table 4 we look deeper into the exact model specifications and predictors that yield the lowest RMSFEs. We start with the results for forecasting CPIall and PCEall inflation using no leads presented in Panel A which summarize the best three models in terms of lowest RMSFEs. We find that these are MIDAS specifications with no quarterly factors given by the ADL-MIDAS model and more precisely by equations 2.3 for $h=1$ and 2.3 for $h=4$ for CPI inflation forecasts. We find that the best daily predictor is Platinum returns at $h=1$ and the A2/P2/F2 Nonfinancial commercial paper spread (with respect to AA Financial Commercial Paper or Federal Funds) at $h=4$. What is particularly interesting is that the top percentile of best predictors for $h=1$ and $h=4$ involve commodity prices and interest rate spreads. Similar results in terms of best predictors and models are found for PCEall at $h=1$ with highest relative forecasting gains over the benchmark compared with those for CPIall. Moreover, while the best predictor for CPIall inflation at $h=1$ is Platinum returns and the best 32 models in terms of lowest RMSFE involve Platinum returns, the second or third best predictors are Reuters Commodity Price Index and the 3Month Tbills based on traditional ADL models, with RMSFE which is almost twice as that of the best ADL-MIDAS models with Platinum returns. Similar results are obtained when forecasting CPIall and PCEall inflation with leads shown in the second half of Panel B in Table 3. Again for both for $h=1$ and 4 the models with the lowest RMSFE are ADL-MIDAS models with no factors given by equation 2.5. Among the best predictors for CPIall with leads we have again the nonfinancial commercial paper spread, some commodity prices as well as the Inflation Compensation for 5 years which features as the best predictor of CPIall inflation using leads at $h=1$. For PCEcore we find that using daily leads of oil prices and the 1 month LIBOR improves forecasts using MIDAS models with no quarterly factors for both 1 
an 4 quarters ahead.

We now turn to the results for the best models and best predictors for forecasting GDP growth found in Table 4 . In Panels A and B we find that the best models for forecasting GDP growth (with no leads) for $h=1$ are quarterly factor ADL-MIDAS models, FADL-MIDAS, given by equation (2.8) with daily Canadian/US Dollar returns as well as the restricted ADL-MIDAS models (with no quarterly factors) with the daily Aruoba, Diebold and Scotti (ADS) indicator given by equation (2.6). For $h=4$ the best predictors of GDP growth are interest rate spreads with the best predictors being the A2/P2/F2 Nonfinancial Commercial Paper spread (minus the AA Financial Commercial Paper) as well as the 1month Eurodollar spread (with respect to the FF) rate. For using daily predictors with leads to forecast GDP growth, we find that mainly short term interest rates (and in particular LIBOR rates of 3months and 1 year) as well as corporate bond spreads of Merill Lynch AA and A ratings, Moody's Baa ratings minus the 10 Year Treasury bond rate are among the best predictors.

Tables 4 and 5 present the results on daily factors and forecast combinations for forecast horizons 1 and 4. Column 1 shows the predictive ability of the daily factor. Column 2 shows the median forecast for the 90 variables of the selected subset while Columns 2 to 7 presents the results for different classes of assets. First of all, we should note that the daily factors perform very well as they can improve forecasts based on the information captured by the quarterly factors and compare favorably against models based on the median forecast, especially when the daily information is aggregated using MIDAS models. Interestingly, while no single class of assets stands out for forecasting IP growth, Equity and Commodities are particularly important for forecasting PCEcore inflation. Furthermore, in the case of PCEcore Inflation ADL-MIDAS and FADL-MIDAS models with leads provide substantial forecasting gains for both the daily factors and the median forecasts. For instance, in the case of the daily factors and $h=4$ the ADL-MIDAS increases forecasting ability by $30 \%$ over the corresponding ADL.

-TO BE COMPLETED-

\section{Conclusion}

In this paper we show that there are substantial forecasting gains for forecasting inflation and economic activity using higher frequency/daily financial data and (F)ADL-MIDAS models 
(F)ADL-MIDAS models improve the forecasts of the benchmarks substantially. For CPI inflation by about $55 \%$ of the RMSFE of the UCSV for both 1 and 4 quarters ahead. For IP by about $70 \%$ and $55 \%$ of the RMSFE of the RW for 1 and 4 quarters ahead, respectively, For GDP growth by about $65 \%$ and $70 \%$ of the RMSFE of the RW benchmark models, respectively, for both 1 and 4 quarters ahead. ADL-MIDAS and FADL-MIDAS models improve the forecasts of conventional ADL models, which are based on flat aggregation. For instance, in the case of CPI Inflation the best ADL-MIDAS improves the accuracy of the best ADL by about $30 \%$.

The set of best predictors varies from variable to variable and forecast horizon. Overall, this set of best predictors includes: For CPI Inflation, (i) Commodities (Platinum, Sugar, WTI Oil Future, Lead Forward, Corn Future, Oat); (ii) Corporate Risk (spread of A2P2F2 with AA Fin CP or AA Non Fin CP, LIBOR, Break-even inflation); (iii) Government Securities (3 Month T-Bill); Foreign Exchange Rates (FXUK, EFXbroad); For GDP growth, (i) Foreign Exchange Rates (Canadian\$/US\$); (ii) Corporate Risk (spread of A2P2F2 with AA Fin CP or AA Non Fin CP, Eurodollar-Fed Funds LIBOR, Break-even Inflation, Merrill Lynch A - 10Y TBond, Merrill Lynch AA - 10Y TBond); (iii) Government Securities (1Y T-Bond Fed Funds); (iv) ADS.

Finally, the daily factors appear to have useful information for forecasting inflation and economic activity beyond the information included in the quarterly factors. MIDAS models can efficiently incorporate this information and provide accurate forecasts, especially when these models incorporate real-time information using daily leads within the quarter. 


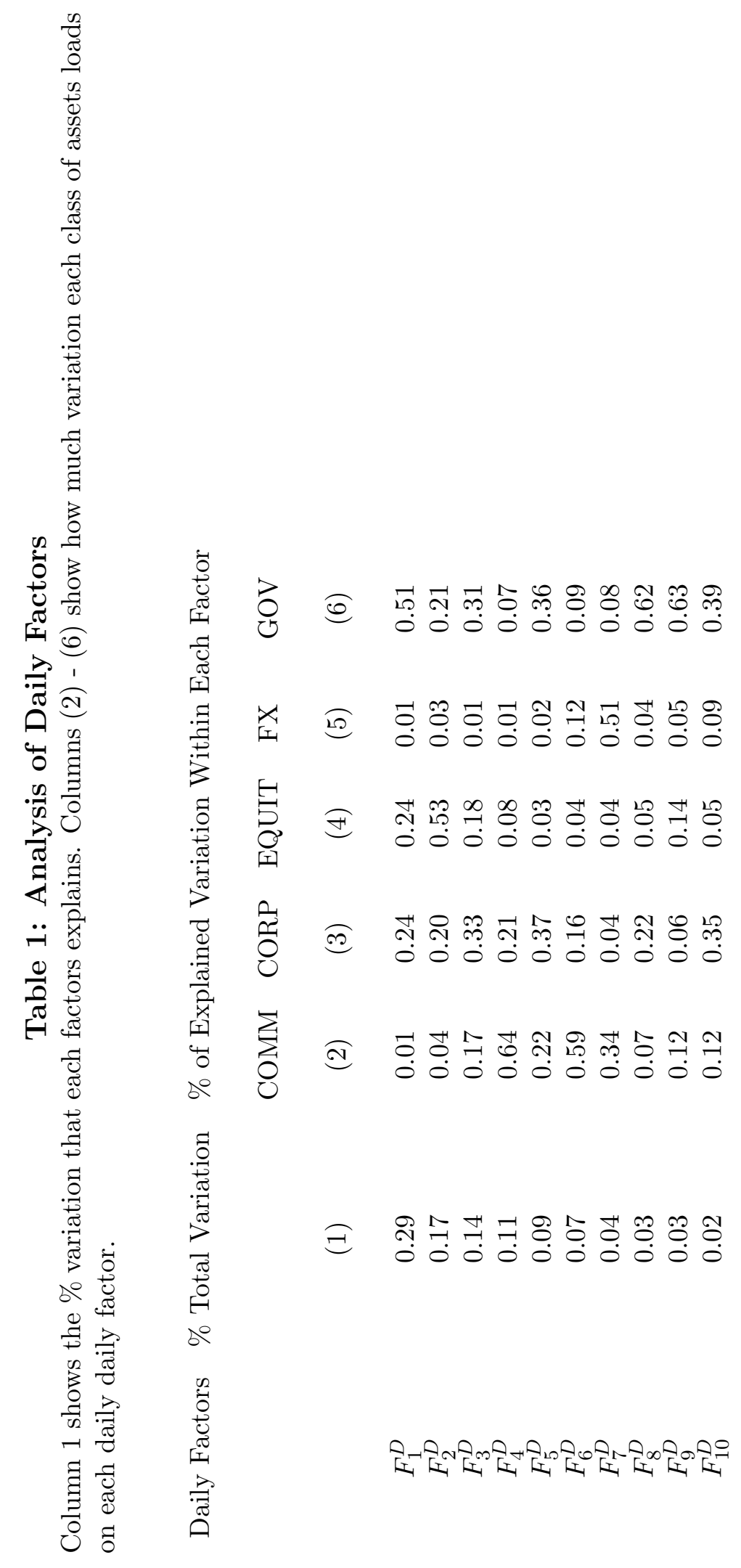




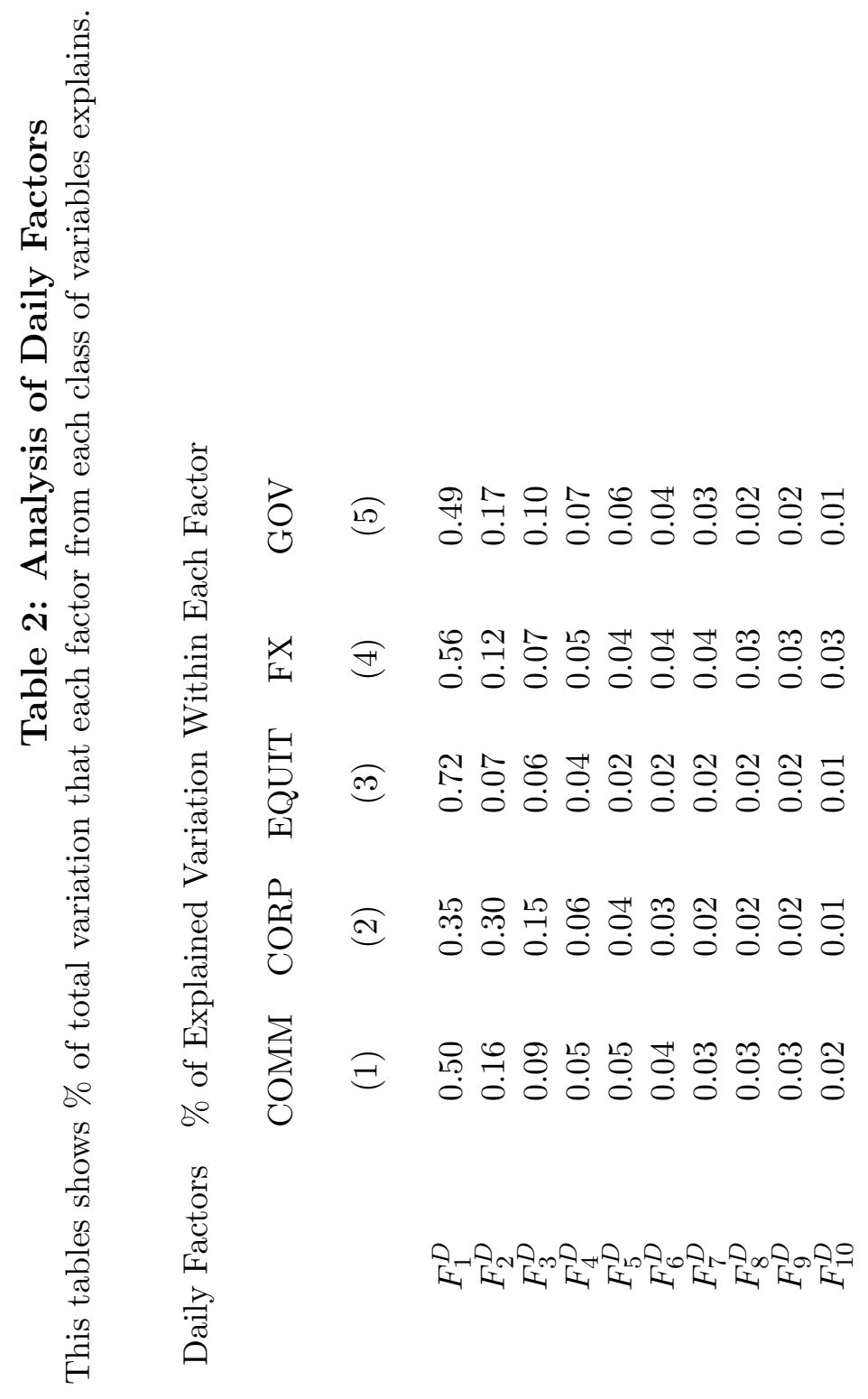




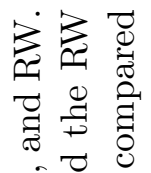

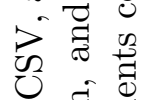

ఏี ฮี

0 范

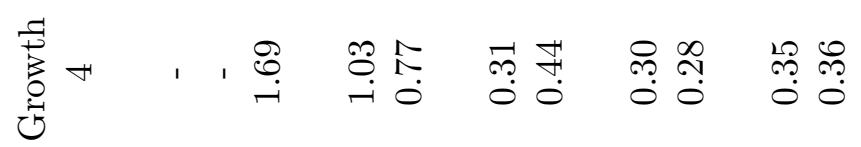

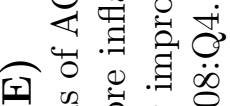

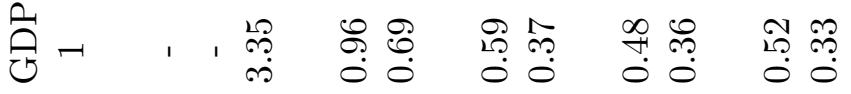

प्र

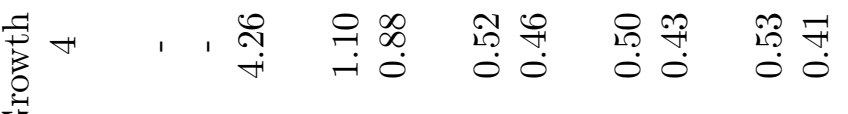

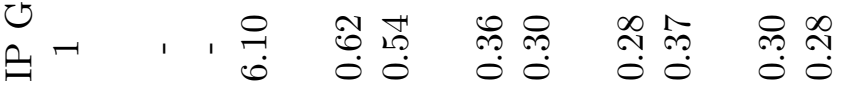

일

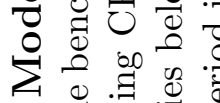

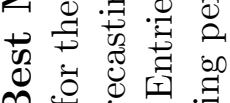

๑

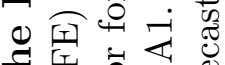

फ

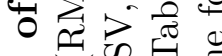

踏记

○

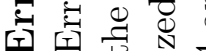

पू

ชै

๑

0ี I $\sum_{0}^{n}$

玒 㐘

․ㅠ.

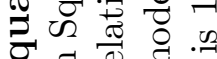

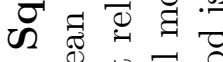

ส $\stackrel{0}{\Sigma}^{0}$

过

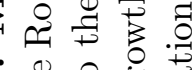

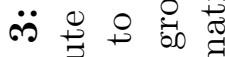

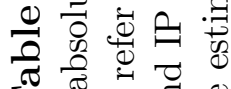

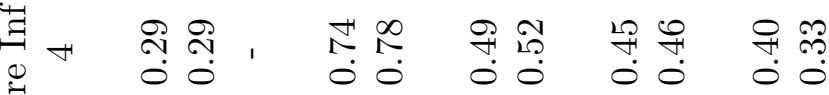

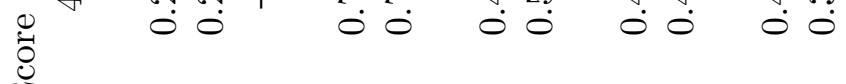

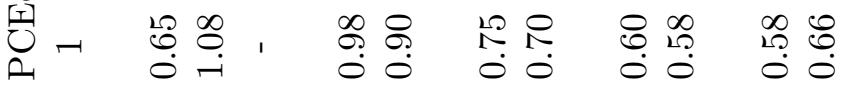

岁

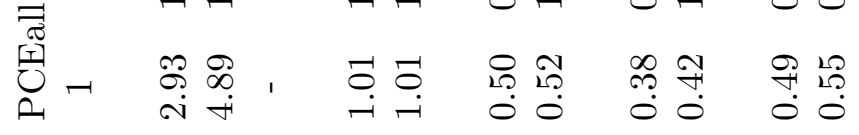

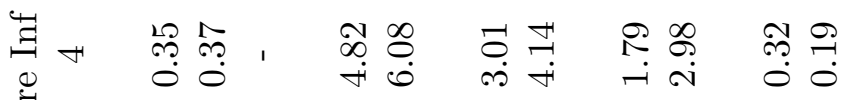

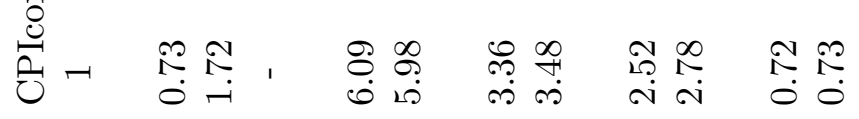

岁

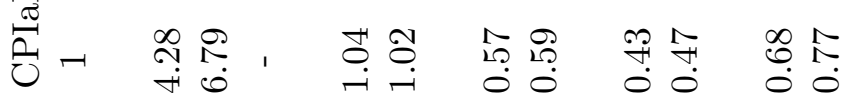

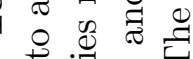

䓽

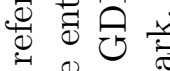

o 50

능

$\vec{n}$ 记

幽

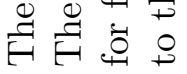

तิ

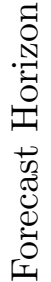

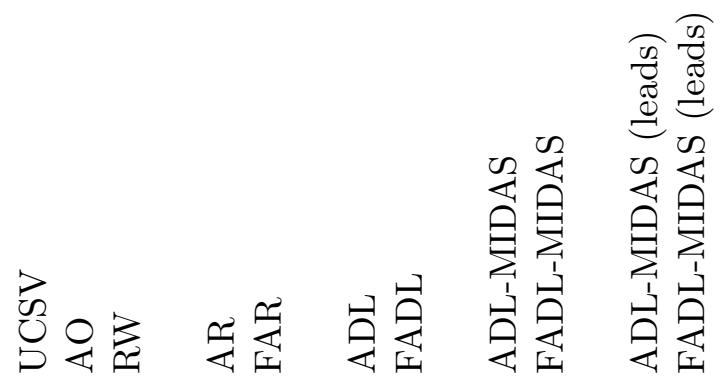




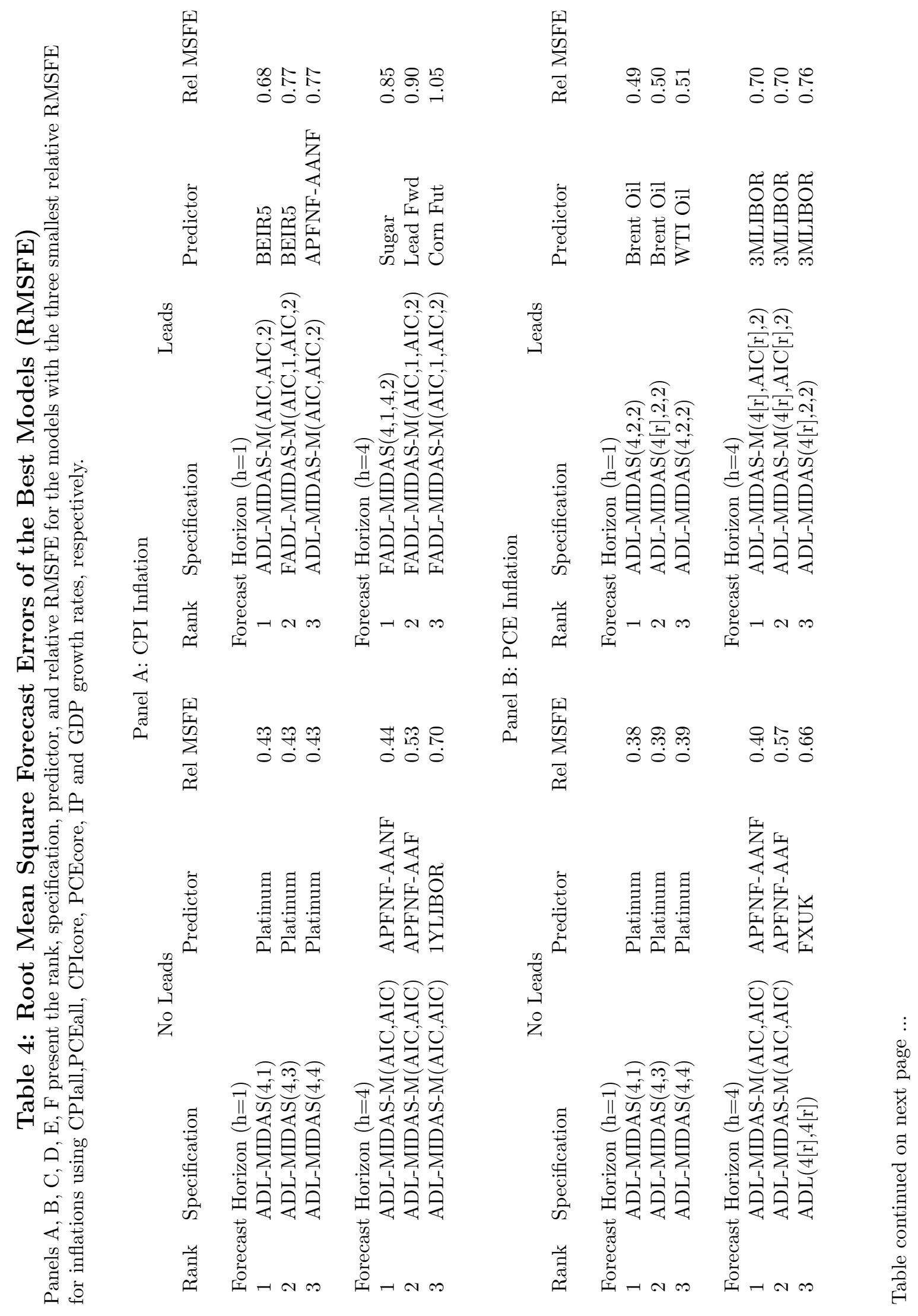




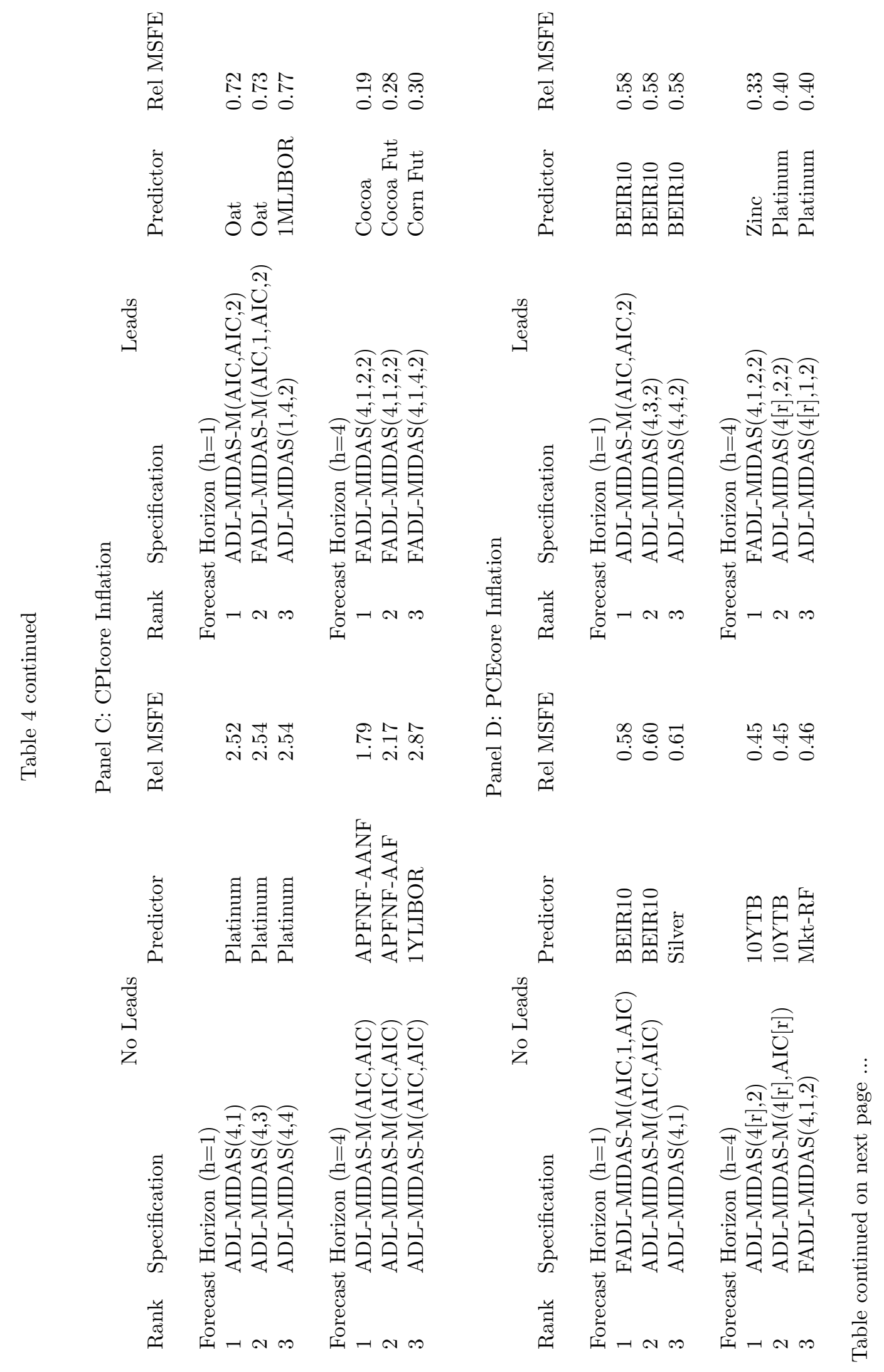




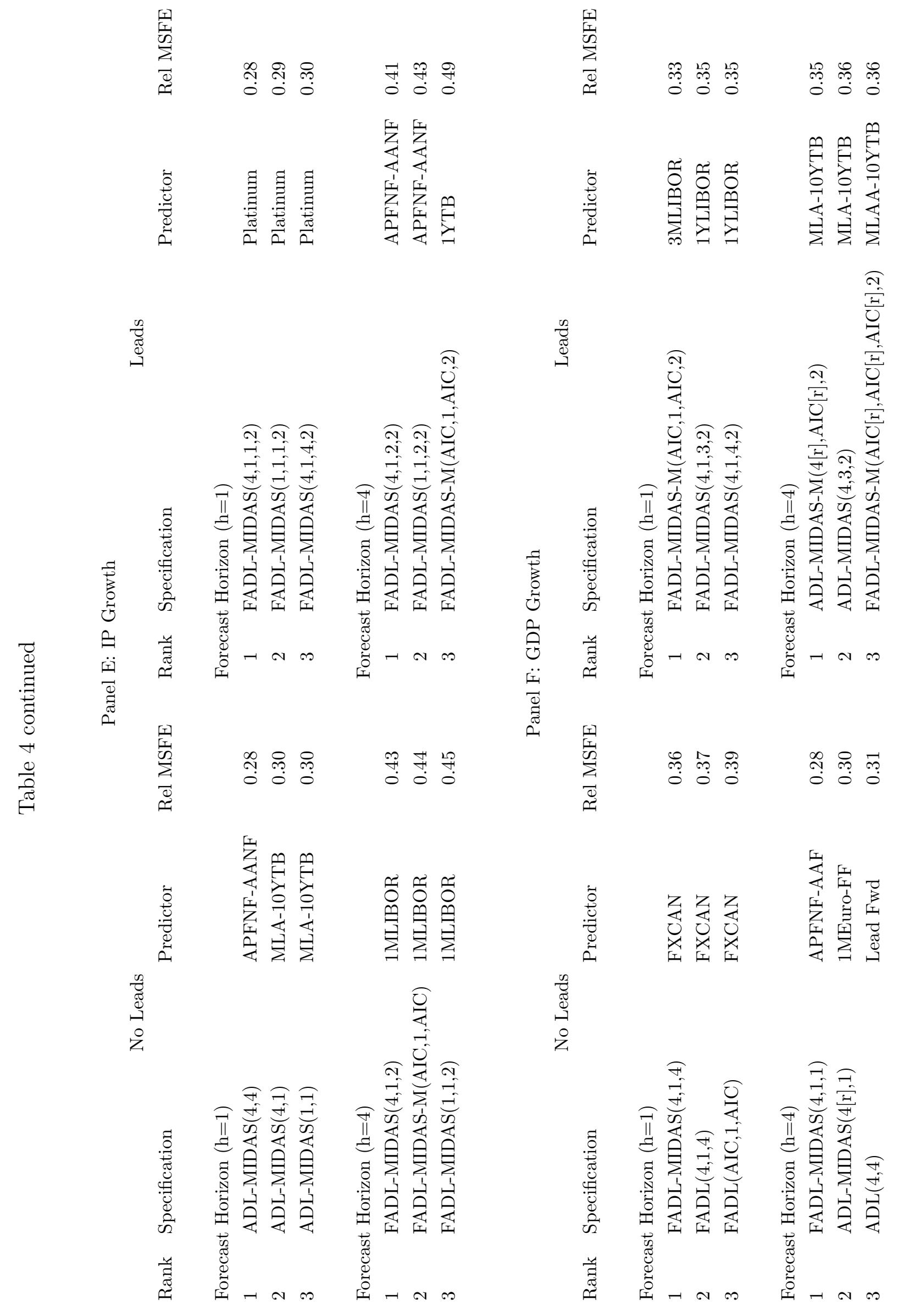




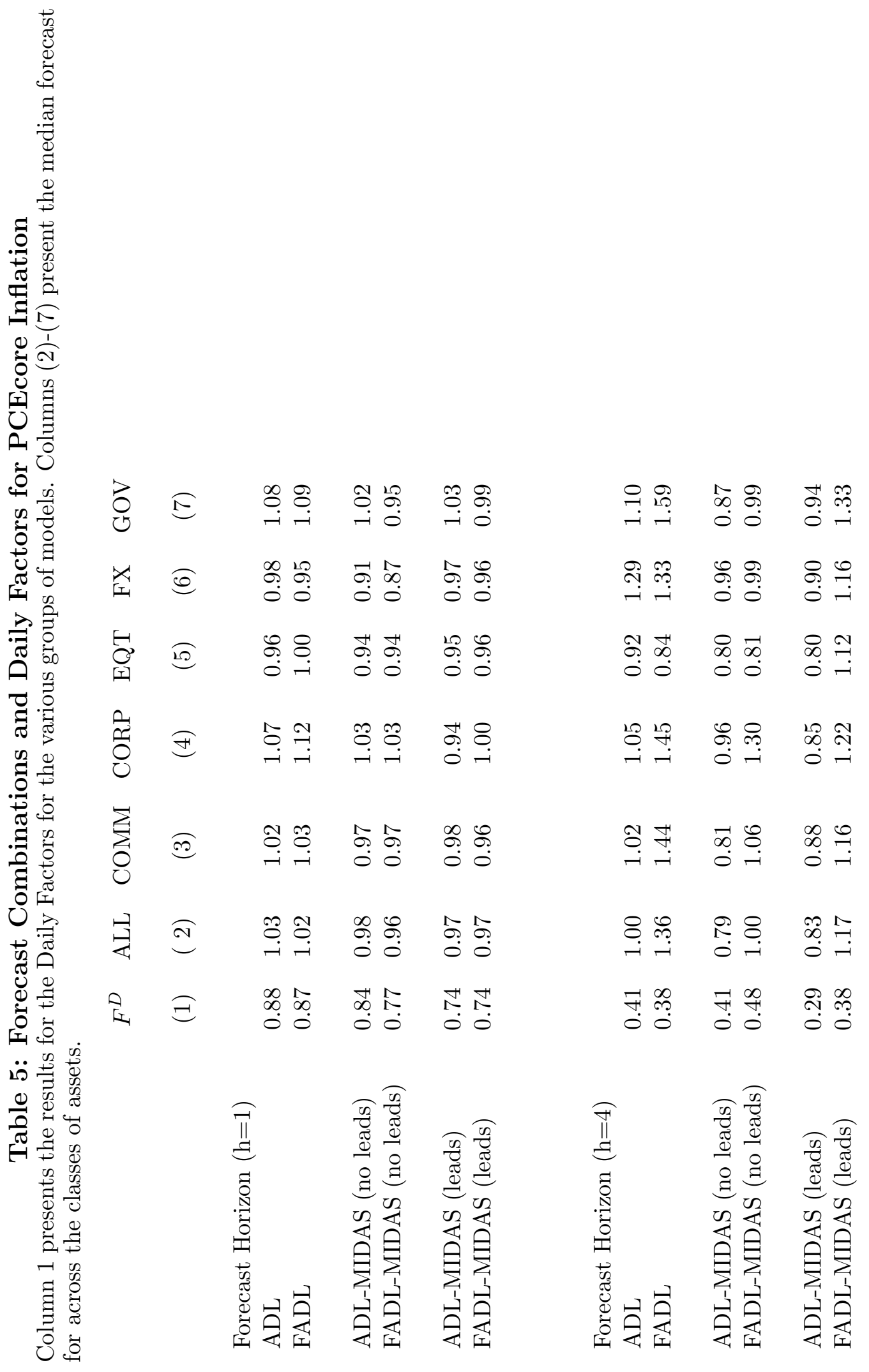




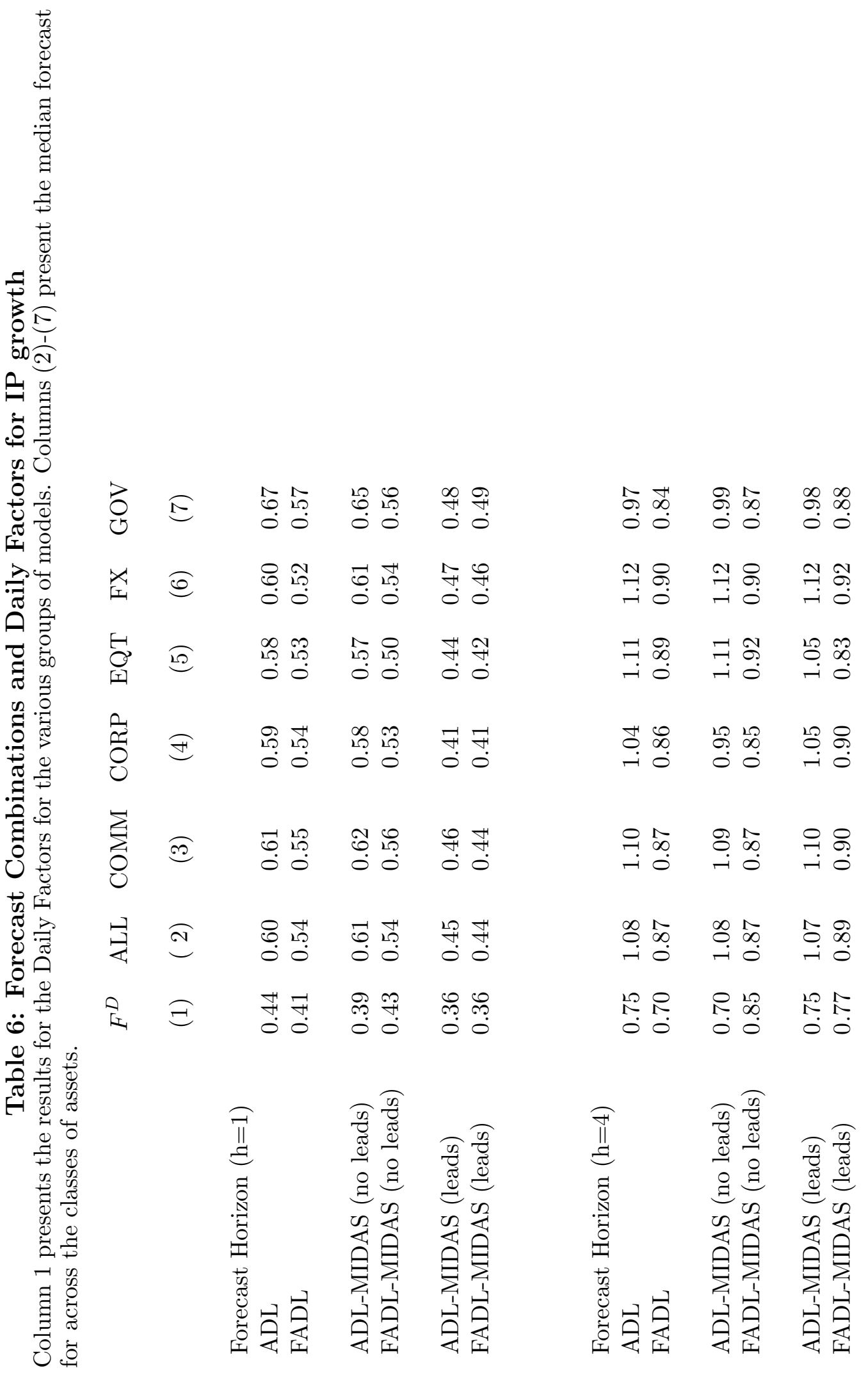




\section{References}

Andreou, E., E. Ghysels, and A. Kourtellos, 2008, Regression Models With Mixed Sampling Frequencies, Journal of Econometrics, forthcoming.

Armesto, M.T., R. Hernandez-Murillo, M. Owyang, and J. Piger, 2008, Measuring the Information Content of the Beige Book: A Mixed Data Sampling Approach, Journal of Money, Credit and Banking (forthcoming).

Aruoba, S.B., F.X. Diebold, and C. Scotti, 2009, Real-time measurement of business conditions, Journal of Business and Economic Statistics (forthcoming).

Bai, J., 2003, Inferential theory for factor models of large dimensions, Econometrica pp. 135-171.

— E. Ghysels, and J.H. Wright, 2009, State Space Models and MIDAS Regressions, Discussion Paper, NY Fed, UNC and Johns Hopkins.

Bai, J., and S. Ng, 2002, Determining the number of factors in approximate factor models, Econometrica pp. 191-221.

Bernanke, Ben, Mark Gertler, and Mark Watson, 1997, Systematic monetary policy and the effects of oil price shocks, Brookings Papers on Economic Activity 1, 91-157.

Chen, X., and E. Ghysels, 2009, News - good or bad - and its impact on predicting future volatility, Discussion Paper, UNC.

Clements, M.P., and A.B. Galvão, 2008a, Forecasting US output growth using Leading Indicators: An appraisal using MIDAS models, Journal of Applied Econometrics (forthcoming).

Clements, M., and A. Galvão, 2008b, Macroeconomic Forecasting with Mixed Frequency Data: Forecasting US output growth, Journal of Business and Economic Statistics 26, $546-554$.

Forni, M., M. Hallin, M. Lippi, and L. Reichlin, 2000, The generalized dynamic-factor model: Identification and estimation, Review of Economics and Statistics 82, 540-554.

— 2003, Do financial variables help forecasting inflation and real activity in the euro area?, Journal of Monetary Economics 50, 1243-1255. 
- 2005, The generalized dynamic factor model, Journal of the American Statistical Association 100, 830-840.

Galvão, A.B., 2006, Changes in Predictive Ability with Mixed Frequency Data, Discussion Paper QUeen Mary.

Ghysels, Eric, Pedro Santa-Clara, and Rossen Valkanov, 2002, The MIDAS touch: Mixed data sampling regression models, Working paper, UNC and UCLA.

— 2006, Predicting volatility: getting the most out of return data sampled at different frequencies, Journal of Econometrics 131, 59-95.

Ghysels, E., A. Sinko, and R. Valkanov, 2006, MIDAS regressions: Further results and new directions, Econometric Reviews 26, 53-90.

Ghysels, Eric, and Jonathan Wright, 2008, Forecasting professional forecasters, Journal of Business and Economic Statistics (forthcoming).

Giannone, D., L. Reichlin, and D. Small, 2008, Nowcasting: The real-time informational content of macroeconomic data, Journal of Monetary Economics 55, 665-676.

Hamilton, J.D., 2006, Daily Monetary Policy Shocks and the Delayed Response of New Home Sales, working paper, UCSD.

Harvey, Andrew, 1989, Forecasting, Structural Time Series Models and the Kalman Filter (Cambridge University Press, Cambridge).

Harvey, Andrew C., and Richard G. Pierse, 1984, Estimating missing observations in economic time series, Journal of the American Statistical Association 79, 125-131.

Kuzin, V., M. Marcellino, and C. Schumacher, 2009, MIDAS versus mixed-frequency VAR: nowcasting GDP in the euro area, Discussion Paper 07/2009 Deutsche Bundesbank.

Mariano, R.S., and Y. Murasawa, 2003, A new coincident index of business cycles based on monthly and quarterly series, Journal of Applied Econometrics 18, 427-443.

Mittnik, S., and P. Zadrozny, 2004, Forecasting quarterly German GDP at monthly intervals using monthly Ifo business conditions data (Springer).

Monteforte, Libero, and Gianluca Moretti, 2008, Real time forecasts of inflation: The role of financial variables, Discussion paper Bank of Italy. 
Schumacher, C., and J. Breitung, 2008, Real-time forecasting of German GDP based on a large factor model with monthly and quarterly data, International Journal of Forecasting $24,386-398$.

Stock, J.H., and M.W. Watson, 1989, New indexes of coincident and leading economic indicators, NBER macroeconomics annual pp. 351-394.

, 2002, Macroeconomic forecasting using diffusion indexes, Journal of Business and Economic Statistics 20, 147-162.

— 2003, Forecasting output and inflation: the role of asset prices, Journal of Economic Literature pp. $788-829$.

— 2008, Forecasting in Dynamic Factor Models Subject to Structural Instability, in Jennifer Castle, and Neil Shephard, ed.: The Methodology and Practice of Econometrics, A Festschrift in Honour of Professor David F. Hendry. Oxford University Press.

Tay, A., 2006, Financial Variables as Predictors of Real Output Growth, Discussion Paper SMU.

Tay, A.S., 2007, Mixing Frequencies: Stock Returns as a Predictor of Real Output Growth, Discussion Paper SMU.

Zadrozny, P.A., 1990, Forecasting US GNP at monthly intervals with an estimated bivariate time series model, Federal Reserve Bank of Atlanta Economic Review 75, 2-15. 


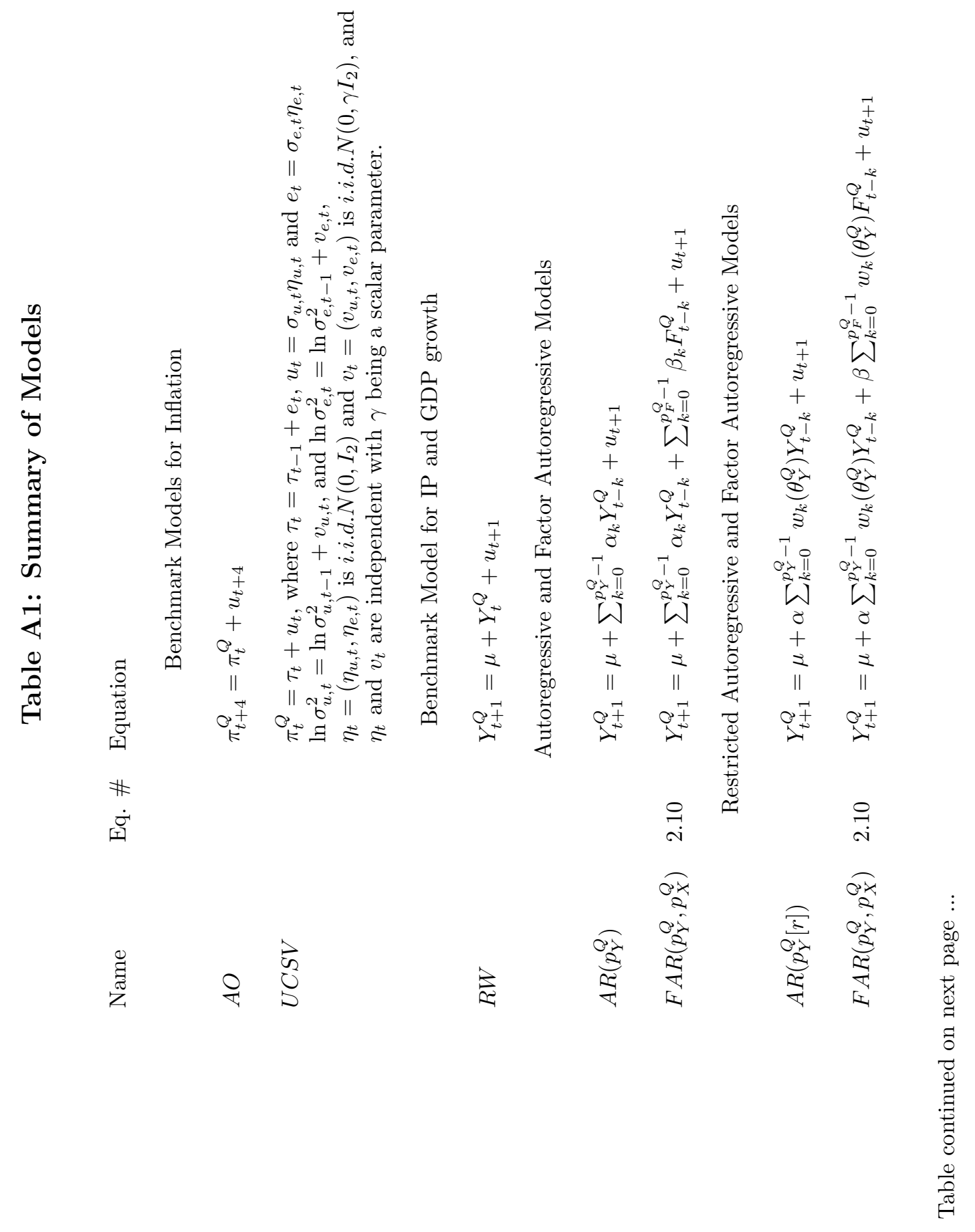



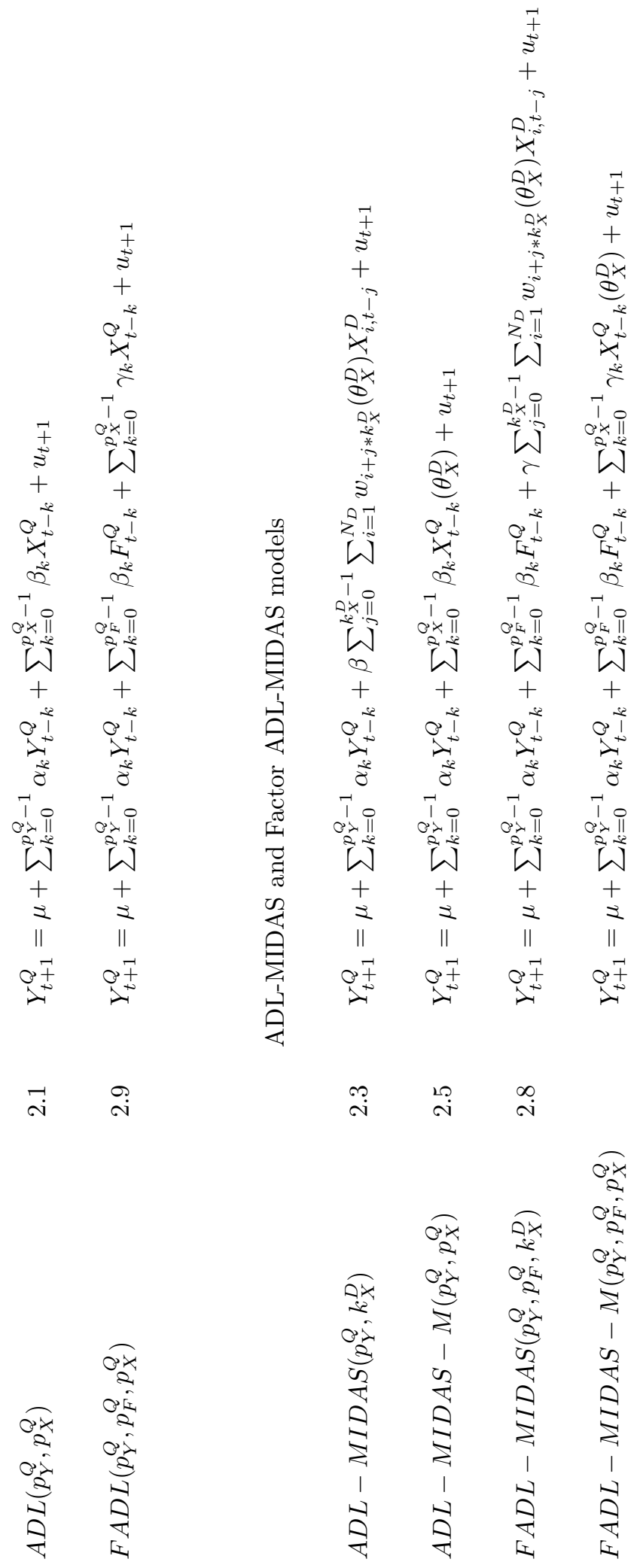


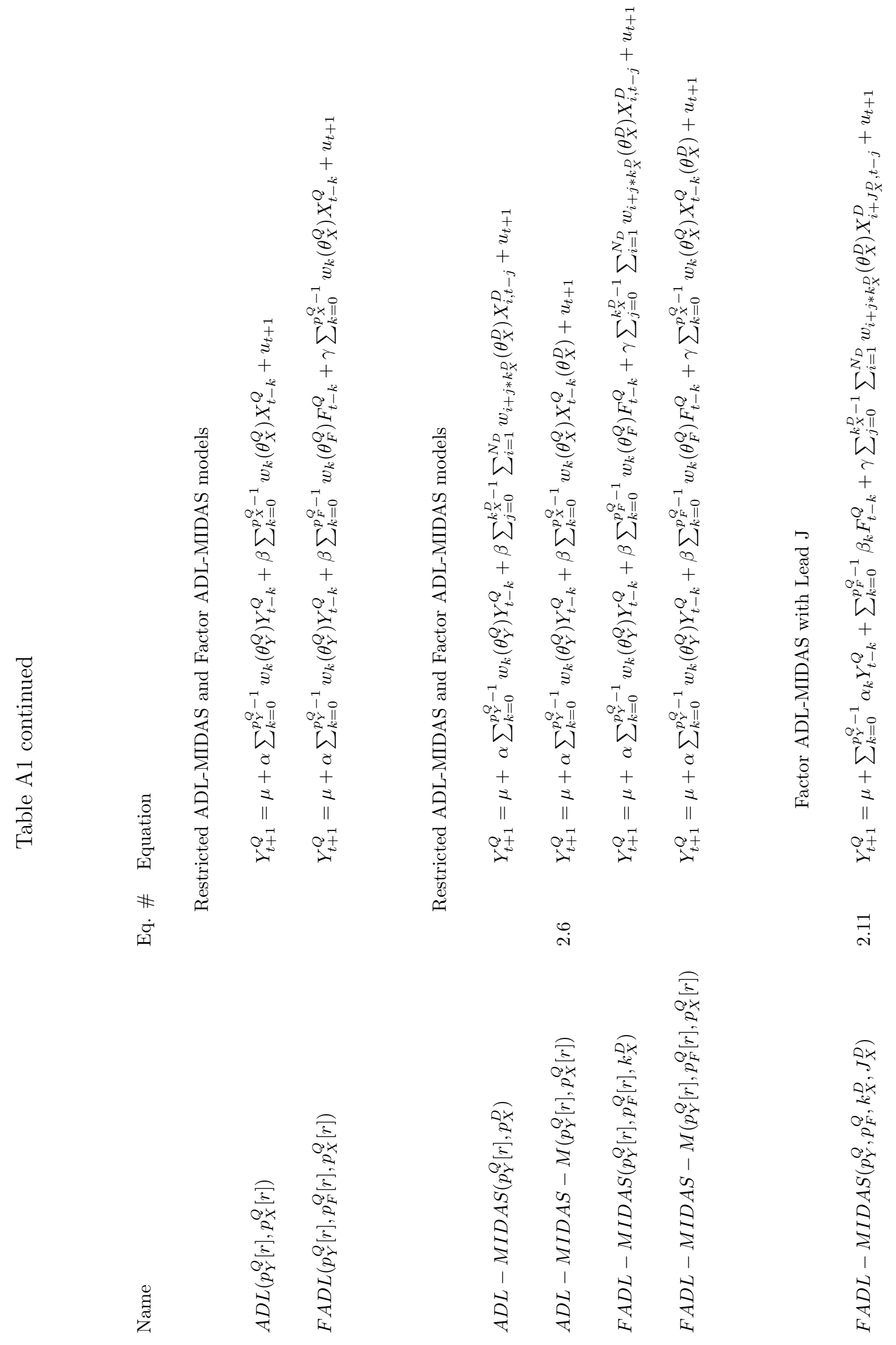

